IV.

Aus dem chirurgisehen Stadtlazareth in Danzig. Oberarzt: Prof. Dr. Barth.

\title{
Ueber drei Fälle von Herznaht wegen Herzverletzung ${ }^{1}$ ).$$
\text { Von }
$$$$
\text { Dr. Wolff, }
$$ \\ Assistenzarzt im Westpreussischen Trainbataillon Nr. 17.
}

Die Behandlung der Verletzungen des IIerzens war noch bis vor wenige Jahre eine rein exspectative und beschränkte sich auf die Anlegung eines Occlusivverbandes, $\Lambda$ nordnung strengster Bettruhe, Eisblase und eventuellen Aderlass, um durch Verringerung des Blutdruckes die Bildung eines die Wunde verstopfenden Thrombus zu erleichtern, wälrend von anderen Aerzten im Gegensatz hierzu Digitalis gegeben wurde. Die früher geltende Ansicht, dass Herzwunden unbedingt tödtlich verlaufen müssten, hatte man schon in den vierziger Jahren des vorigen Jahrhunderts aufgegeben, und $\mathrm{Fischer}{ }^{2}$ ) stellte im Jahre 1868 als erster eine vortreffliche Statistik aller Heilungen nach Herzverletzungen auf, in der er die Mortalität auf 90 Proc. berechnete. $\mathrm{Ihm}$ verdanken wir auch die Untersuchungen über die Todesursachen nach Herzverletzungen, wenngleich die wesentlichsten Aufschlüsse hierüber erst $\operatorname{Rose}^{3}$ ) brachte. In seiner Arbeit über die „Herztamponade" nahm er als häufigste Todesursache die allmäbliche Erdrückung des Herzens durch die im Herzbeutel eingeschlossene und aus der Herzwunde stetig zunchmende Blutmenge an und verwies damit die Behandlung der Herzverletzungen auf das Gebiet der Chirurgie. Seitdem wir dann in die aseptische Aera eingetreten waren, wurde auch von einzelnen Chirurgen die Möglichkeit einer Operation in's Auge gefasst; den letzten Schritt in dieser Richtung

1) Vgl. Wolf f, LeberHerznaht und Herzverletzungen. Inaug.-Diss. Leipzig 1902.

2) Fischer, Archiv f. klin. Chirurgie. 1868.

3) Rose, Herztamponade. Deutsche Zeitschr. f. Chirurgie. Leipzig 1534. 
thaten Del Vecehiol) und nach ihm Salomoni2), die durch ihre Versuche an Hunden die Ausführbarkeit von Operationen am Herzen darthaten. Im Herbst des Jahres 1896 endlich veröffentlichten Farin $a^{3}$ ) und $\operatorname{Reh}^{3}$ ) ziemlich gleichzeitig die ersten Fälle von Herznaht am Menschen. Seitdem ist bereits eine ganze grosse Literatur der Herzchirurgie entstanden und ich habe aus ihr nicht weniger als 39 Fälle von Herznaht zusammenstellen können. Wenn ich trotzdem jetzt 3 weitere, bisher noch nicht bekannte Fälle veröffentliche, so geschieht das; weil ich meine, dass bei der für die Häufigkeit der Verletzung immerbin noch geringen Casuistik jeder einzelne Fall hohes Interesse beanspruchen darf. Ferner aber auch deshalb, weil mir bei der grossen Variabilität der Herzverletzungen gerade aus diesen Fällen gewisse einheitliche Gesichtspunkte sich zu ergeben scheinen. Die Fälle sind mir durch die Liebenswürdigkeit des Professor Barth, Oberarztes am städtischen Krankenhause Sandgrube in Danzig, zur Verfügung gestellt und betreffen 3 Leute, von denen er 2 im April 1901, 1 im Februar 1902 operirt hat.

Der erste Fall betrifft einen 28 jährigen Mann, der eine halbe Stunde vor Aufnahme in das Krankenhaus einen Stich in die Magengrube erhielt, noch 20 Schritt weit gehen konnte und dann bewusstlos zusammenbrach. Er wurde von Collegen sofort in das Lazareth Sandgrube gebracht. Hier ergab die Untersuchung: Patient ist ein Mann von kräftiger Musculatur, er ist völlig apathisch und antwortet anf an ihn gerichtete Fragen nicht, sondern stöhnt nur fortwährend. Der Puls ist kanm futhlbar, die Herztöne sebr leise, die Gesichtsfarbe todtenblass. Eine Lungenuntersuchung wird des schweren Zustandes des Verletzten wegen nicht vorgenommen. In der Magengrube befindet sich ein etwa $2 \mathrm{~cm}$ langer, etwas schräg gestellter Stich, welcher durch den Processus ensiformis führt. Nach Erweiterung der Wunde zeigt sich, dass der Stich nicht, wie vermuthet, in die Bauchhöhle, sondern nach oben unter das Brustbein futhrt und rechte Pleura sowie Herzbentel eröffnet hat, sodass Luft ausund einströmt. Die Pulsation des Herzens wird direct unter dem Finger gefuhlt. Nach vorläufiger Tamponade werden die Knorpel der 5., 6. und 7. Rippe rechts vom Sternum in einer Ausdehnung von je $2-3 \mathrm{~cm}$ resecirt und, da der Herzbeutel auch jetzt noch nicht genügend zugänglich ist, der Processus ensiformis nebst einem $3 \mathrm{~cm}$ langen Stück des Corpus sterni bis zum Ansatz der rechten Rippenknorpel fortgenommen. Nun erkennt man einen blutenden Riss in der rechten Pleura, der zunächst tamponirt wird, sowie eine etwa $2 \mathrm{~cm}$ lange, mässig blutende Perikard-

1) Del Vecchio, Sutura del cuore. Ref. Centralbl. f. Chirurgie. 1895.

2) Salo-moni, XI. Congress der ital. Gesellschaft für Chirurgie. Rom 1896 Desgl. Farina.

3) Rehn, Penetrirende Wunden und Suturen des Herzens. Archiv f. klin. Chirurgie. Berlin 1897. 
wunde, die nach links hin quer erweitert wird, sodass das Herz frei liegt. Aus dem Herzbeutel werden 2 kleinfaustgrosse Blutcoagula extrahirt. Jetzt wird im rechten Ventrikel, etwa $4 \mathrm{~cm}$ oberhalb der Spitze und 2-3 cm von der Coronaraterie entfernt, eine etwas schräg gestellte, $2 \mathrm{~cm}$ lange und wenig klaffende Stichwunde sichtbar, aus der beim Anheben wenig Blut quillt. Mit einiger Mühe wird eine Seidennaht durchgefthrt und, nachdem das Herz an dieser besser in die Wunde eingestellt ist, werden mit Leichtigkeit 3 weitere Seidennähte gelegt, sodass die Wunde exakt geschlossen ist. Naht der Pleura und Perikardwunde bis anf eine $1 \frac{1}{2} \mathrm{~cm}$ lange Oeffinuug, in welche ein dunner Jodoformgazestreifen eingefuhrt wird. Tamponade der ubrigen Wunde, theilweise Hautnaht. Der Puls ist sofort nach Ausräumung des Herzbeutels wieder fuhlbar geworden und beträgt jetzt 72, ist mässig gespannt. Es sind während der ganzen etwa eine Stunde dauernden Operation nur einige Züge Chloroform verabreicht und da Patient nicht reagirte, so ist im Uebrigen ohne Narkose operirt worden.

Am folgenden Tage beträgt der Puls 140 Schlăge, ist von guter Spannung. Temperatur 38,4. Patient klagt aber viel tber Angstgefuhl und Athemnoth. Er wird hochgelagert. Der Verband ist stark blutig durchtränkt und wird deshalb in seinen oberen Schichten gewechselt. Am nächsten Tage sind die subjectiven Beschwerden geringer. Puls 120. Temperatur 39,2. Nach Entfernung des im Perikard liegenden Tampons ergiesst sich sehr viel blutig seröse Flussigkeit aus dem Herzbeutel. Es wird daher ein Gummidrain eingefuhrt. Patient erhält eine Kochsalzinfusion. Vom nächsten Tage ab entwickelt sich eine beiderseitige Pleuritis; eine Punction der rechten Pleura ergiebt ein aseptisches, blutiges Exsudat. Am 4. Tage nach dor Operation tritt unter zunehmender Athemnoth und Herzschwäche der Tod ein.

Gerichtlicher Sectionsbefund: Der Herzstich ist perforirend, er hat einen starken Trabekel vollständig und den vorderen Zipfel der rechten Atrioventricularklappe zur Hälfte durchschnitten. Die Wundränder liegen gut an und sind bereits fest miteinander verklebt. Der ganze Herzbentel ist mit dicken Fibringerinnseln ausgefullt. Die Lungen zeigen beiderseits geringe pleuritische Verwachsungen, im rechten Pleuraraum finden sich Blutgerinnsel und flussiges Blut, im linken ein entzilndlicher, hämorrhagischer Flussigkeitserguss.

Der $\mathrm{zw}$ eite Fall betrifft einen 28 Jahre alten, kraftig entwickelten Mann, der zwei Tage nach dem ersten Falle in's Krankenhaus Nachts eingeliefert wurde, nachdem er laut Angabe seiner Begleiter vor $3 / 4$ Stunden auf dem Closett eines Restaurants mit Stichen in der Herzgegend aufgefunden war, die er sich selbst beigebracht hatte.

Patient, der auf Fragen schwache Antworten giebt, ist sehr blass, die Lippen weiss, die Athmung äusserst muhsam. Im 4. linken Intercostalraum befinden sich, $3 \mathrm{~cm}$ vom linken Sternalrande entfernt, 3 je $1 / 2 \mathrm{~cm}$ lange Stichwunden, aus denen eine Blutung nicht mehr stattfindet. Der Puls ist nicht fuhlbar, die Herztöne sind sehr leise, Spitzenstoss nicht fuhlbar. Es besteht linksseitiger Pneumothorax; die Athmung ist flach und beschleunigt.

Nunmehr wird in Chloroformnarkose ein hufeisenförmiger Schnitt - 
ähnlich dem Rydygier'schen I) Hautschnitt - gemacht, der vom Ansatz der 4. Rippe am rechten Sternalrande ausgehend quer uber das Sternum nach links ther die Knorpelknochengrenzen der 4. und 5. linken Rippe verläuft und unterhalb des 5. Rippenknorpels wieder uber das Sternum bis zum rechten Rande zurückgeht. Die Knorpel der 4. und 5. linken Rippe werden resecirt, das etwa $3 \mathrm{~cm}$ lange Sternalstilck durchschnitten und nach rechts hinübergeklappt. Nach Eröffoung des Brustkorbes findet sich eine Stichwunde in der Pleura, die eine geringe Blutung zeigt, und sofort genäht wird. Der Herzbeutel weist eine $1 / 2 \mathrm{~cm}$ lange Stichwunde auf; nach seiner Eröffnung durch einen etwa $5 \mathrm{~cm}$ langen Schnitt finden sich grosse Sticke geronnenen Blutes und reichlich flüssiges Blut, nach dessen Entfernung der Puls sofort wieder futhlbar wird. Darauf wird ein $1 / 2 \mathrm{~cm}$ langer, längs verlaufender Stich im linken Ventrikel, $1 \mathrm{~cm}$ von. der Cornpararterie entfernt, sichtbar, aus dem eine Blutung nicht mehr stattfindet. Nachdem das Herz mit flachen, krummen Haken nach vorn und rechts gedrängt worden ist, werden 3 Seidennähte, die das Endokard nicht mitfassen, hindurchgelegt. Eine deutliche Unterscheidung zwischen Systole und Diastole ist, solange das Herz mit den Haken gefasst ist, nicht zu machen. Darauf Naht der Stichwunde des Perikards mit Catgut und vollständiger Versehluss der Operationswunde des Herzbeutels mit Catgutnähten. Hautnaht bis zum unteren Wundwinkel, aus welchem ein Jodoformgazestreifen herausgeleitet wird. Kochsalzinfusion.

In den nächsten beiden Tagen bestehen noch Temperaturen bis $39^{\circ}$, der Puls ist klein und frequent, 120, die Athmng beschleunigt. Die subjectiven Beschwerden sind gering. Vom 3. Tage ab wird der Puls ruhiger und kräftiger, Patient ist fieberfrei. Nach 14 Tagen wird die Weichtheilwunde zur schnelleren Heilung secundär genäht. Patient steht nach 3 Wochen, nachdem er in Folge einer schnell abklingenden rechtsseitigen trockenen Pleuritis noch einmal 2 Tage gefiebert hat, auf. Er hat keine Athem- oder Herzbeschwerden, der Puls beträgt $80-90$ Schläge in der Minute, am Herzen sind Gerăusche nicht wahrnehmbar. 34 Tage nach der Operation wird er geheilt entlassen.

Ein halbes Jahr später, im November 1901, wurde er gelegentlich eines Vortrages von Professor B arth ${ }^{2}$ ) in der Naturforschenden Gesellschaft in Danzig vorgestellt. Er klagte damals tiber geringe Beschwerden beim Bücken, sein Puls war ruhig und regelmässig, die Herztöne rein. Im Gefängniss, wo er sich zur Zeit befand, wurde er mit leichterer Arbeit beschäftigt. Ueber sein weiteres Schicksal nach der inzwischen erfolgten Entlassung aus dem Gefängniss ist nichts zu erfahren.

Der dritte Fall Barth's endlich gelangte am 23. Februar 1902 zur Aufnahme und betraf einen 22 Jahre alten Arbeiter, der vor einer Stunde einen Messerstich in die linke Brust bekommen hatte, noch eine Treppe hinuntergegangen war und dann ohnmächtig zusammenbrach. Als er in's Krankenhaus gebracht wurde, hatte er das Bewusstsein wiedererlangt.

1) Rydygier, Ueber Herzwunden. Wiener klin. Wochenschr. 1898. Nr. 47.

2) Barth, Die Chirurgie des Herzens. Schriften der Naturforschenden Gesellschaft in Danzig. N. F. 10. Bd. 4. Heft. 1902. 
Die Untersuchung ergab: Es handelt sich um einen kräftigen Mann, der sehr blass aussieht, völlig apathisch ist, aber auf an ihn gerichtete Fragen zu antworten vermag. Der Pals ist mittelkräftig, 92 Schläge in der Minute, die Athmung nicht sehr beschleunigt. $3 \mathrm{~cm}$ nach innen und oben von der linken Mammilla beginnt eine sich schräg abwärts nach dem Proc. ensiformis zu hinziehende $4 \mathrm{~cm}$ lange Wunde mit scharfen Rändern, aus der kein Blut sickert. Die Untersuchung des Herzens ergiebt leise Töne und geringe Verbreiterung der Herzdämpfung, sonst nichts Anormales; die Untersuchung der Lungen ergiebt Pneumothorax links. Da der Verdacht auf Herzstich vorliegt, wird die Wunde erweitert, Man findet den Knorpel der 5. Rippe durch-und den der 6. Rippe angeschnitten. Fauchend tritt Luft aus der Wunde aus. Die Pleura parietalis findet sich zwischen den Rippen durchschnitten, aus der Wunde entleert sich nur wenig Blut. Nachdem von den verletzten Knorpeln je ein $1 \frac{1}{2} \mathrm{~cm}$ grosses Sttlck resecirt ist, wird die Pleura zur besseren Uebersicht breit eröffnet. Die Art. mammaria int., die angeschnitten gefunden wird, wird unterbunden und durchschnitten. Nach Eröffnung der Pleura sieht man eine $3 / 4 \mathrm{~cm}$ lange Schnittwunde im Herzbeutel, aus der dickes, flüssiges Blut quillt. Der Puls, der während der letzten Manipulationen sehr schlecht geworden war, erholt sich sofort wieder, als nun der Herzbeutel breit von der Wunde aus eröffnet wird. Nach Entfernung des im Herzbeutel reichlich vorhandenen Blutes findet man in der rechten Kammer etwa $1 \frac{1 / 2}{\mathrm{~cm}}$ von der Grenze zwischen Vorhof und Ventrikel nach unten und $3 \mathrm{~cm}$ seitlich vom Septum eine $3 / 4 \mathrm{~cm}$ lange, scharfrandige Wunde, aus der reichlich Blut während Systole und Diastole quillt. Das Herz pulsirt kräftig, um nur dann unruhig zu zucken, wenn ein Instrument es berührt. Durch 5 Nähte wird die Wunde des Herzens geschlossen. Der Herzbeutel und die Pleura werden ebenfalls primär genäht. Theilweise Naht der Weichtheilwunde, einschliesslich der Musc. interossei. Ein Tampon wird zwischen die Weichtheile bis an das Perikard eingeschoben und nach aussen geleitet.

Die Operation hatte genau eine Stunde gedauert, die Narkose war dauernd gut gewesen. Nach dem Transport in's Bett beträgt der Puls 100 Schläge, ist regelmässig, etwas weich; die Athmung ist sehr mühsam, ihre Frequenz 38.

In den nächsten beiden Tagen klagt Patient beständig über heftige Athemnoth, die Frequenz schwankt zwischen 36 und 40 Athemzilgen, die Pulsfrequenz zwischen $112-128$. Fieber besteht nicht. Vom 2. Tage an setzt ein beinabe $1 / 4$ stundiges Erbrechen ein, das den Patienten ausserordentlich mitnimmt und bis zum 4. Tage anhält. Abgesehen davon hat er keine Beschwerden, die Wunde beilt reactionslos. Die Herztöne sind rein, weder perikardiales, noch pleuritisches Reiben irgendwo hörbar. Nach einigen Tagen allgemeinen Wohlbefindens tritt ein quälender Husten auf, es entwickelt sich eine rechtsseitige Pleuritis mit blutig serösem Exsudat, das am 10 . Tage bis $3 \mathrm{~cm}$ oberhalb des Schulterblattwinkels steht und mehrmals entleert wird. Der Puls bleibt dauernd gut, etwa 80 Schläge in der Minute. Am 17. Tage steht Patient zum ersten Male auf; er fuhlt sich, trotzdem das Exsudat noch bis zum Schulterblattwinkel reicht, völlig wohl, hat keinerlei Schmerzen mehr und hustet nur noch wenig. 
Bei seiner Entlassung in der 5. Woche ist die äussere Wunde fest vernarbt. Patient hat nur noch zeitweise schnell vorübergehende Anfälle von Herzklopfen und Athemnoth, fuhlt sich aber im Uebrigen wohl. Herztöne rein, Puls 82 . Hinten rechts besteht uber den abhängigen Lungenpartieen noch handbreite Dämpfung.

Patient, der augenblicklich in einem anderen Orte im Gefängniss sitzt, wurde Mitte November auf meine Veranlassung ärztlich untersucht. Danach fühl er sich, bis auf kurze, alle 2-3 Wochen auftretende Anfälle von Athemnoth, völlig wohl und kann leichte Arbeit verrichten. Die Herztöne sind rein, unmittelbar ther der Narbe ist bei der Athmung ein leises, aber deutliches schabendes Geräusch zu hören. Puls regelmässig und kräftig, 84 Schläge in der Minute.

Bevor ich auf eine Würdigung der soeben beschriebenen Fälle und damit auf die zu behandelnden Fragen, wann, weshalb und wie operiren wir bei Herzverletzungen, näher eingehe, will ich erst die übrigen 39 Fälle von Herznaht nennen, die ich aus der Literatur gesammelt habe ${ }^{1}$ ).

Es sind:

1. Farina (l. e.): Stichwunde des linken Ventrikels. 3 Nähte. Tod nach mehreren Tagen in Folge Pleuritis und Pneumonia.

2. Rehn (l. c.): Am 2. Tage nach der Verletzung Naht der Stichwande des rechten Ventrikels. Naht des Perikards. Heilung.

3. Cappelen ${ }^{2}$ ): Nicht penetrirende Stichwunde des linken Ventrikels genäht. Tod am 3. Tage in Folge starker Blutung aus der übersehenen Verletzung eines Zweiges der Coronararterie und in Folge von Perikarditis.

4. amoni $^{3}$ ): Im rechten Ventrikel 2 Stichwunden. Sehr schwierige Naht wegen starker Blutung. Heilung.

5. Parlavechio ${ }^{4}$ ): $3^{1 / 2} \mathrm{~cm}$ lange, $V$-förmige Stichwunde des linken Ventrikels. Völlige Heilung nach Naht.

6. Parozzani5): Stichwunde an der Herzspitze, für den kleinen Finger durchgängig, genäht. Perikard primär genäht. Heilung.

7. Parrozzani: Stichwunde des linken Ventrikels. Naht ohne Narkose. Tod am 2. Tage in Folge linksseitiger Pleuritis und starker Anämie.

1) Einige mir zweifelhafte Fälle von Herznaht habe ich weggelassen, so einen bei Podrez erwähnten: La chirurgie du coeur. Revue de Chirurgie. 1899. 19. Bd., der von Parazzavi operirt sein soll und wohl mit dem ersten Fall von Parozzani identisch ist; desgl. einen von Tassi, Bulletino della Academia medica di Roma 1896/97 veröffentlichten Fall Capello, der wohl mit dem Cappelen's derselbe ist.

2) Cappelen, Deutsche med. Wochenschr. Literaturbeilage. 1896.

3) Pamoni, Riforma medica. 1898. Ferner Monatsschr. f. Unfallheilk. 1899.

4) Parlavechio, Riforma medica. 1898. Ref. Handb. d. prakt. Chirurgie 1898. III.

5) Parozzani, Bull della Academia medica di Roma. 1896/97. Ref. Centralbl. f. Chirurgie. 1898. 
8. Marion 1): Revolverschuss. Einschuss im rechten Ventrikel. Naht. Unmittelbar danach Tod. Sectionsbefund ergiebt Ausschussöffinung an der Hinterseite des rechten Ventrikels, Zerreisung der Tricuspidalklappe.

9. Kosinki ${ }^{2}$ ): Stichwunde des rechten Ventrikels. Naht. Heilung.

10. Horodynski 3 ): Stichwunde des rechten Ventrikels, nahe am Septum. Nalit. Perikard primär vernäht. Tod nach 3 Wochen an beiderseitiger eitriger Pleuritis.

11. Mal i s zew ski: Stichwunde des linken Ventrikels. Naht. Perikard drainirt. Tod am 4. Tage in Folge eitriger Perikarditis.

12. Maliszewski: Lappenförmige Stichwunde an der Herzspitze. Beim Versuch ihn anzunähen reisst er ab. Trotzdem Verschluss durch Naht. Perikard vernäht. Resection der verletzten Lungenpartieen. Tod am folgenden Tage in Folge hochgradiger Anämie.

13. Carnobel 1): Stichwunde an der Herzspitze. Catgutnaht. Tod am 5. Tage in Folge putrider Pleuritis.

14. F on $\tan ^{5}$ ): Stichwunde des linken Ventrikels genäht. Catgutnaht des Herzbeutels und der Pleura. Heilung.

15. Fontan 6 ): Stichwunde oberhalb der Spitze. Naht. Perikardnaht. Am 6. Tage Pneumonie des rechten Unterlappens. Später Phlebitis. Heilung.

16. Williams ${ }^{\top}$ ): Stichwunde des linken Ventrikels, nicht penetrirend. Naht. Heilung.

17. Walkers): In der Vorderwand des linken Ventrikels 8 Stichwunden. 11 Seidenuähte. Herzbeutel an äusserer Haut befestigt. Abends starke Lungenblutung. Tod am folgenden Tage. Lunge völlig atelektatisch, Pleura und Perikard mit eitrigen Auflagerungen.

18. Walker: Stich nnter den linken Rippenbogen. Wunde in der Spitze des rechten Ventrikels. Nähte schneiden wiederholt durch und haften erst, nachdem ein Gazetupfer eingebunden ist. Tod nach einer halben Stunde an Verblutung.

19. Giordanos): Stark blutende Stichwunde am linken Herzohr. Perikard-Myocardnähte. Tod am 19. Tage in Folge eitriger Pleuritis.

1) Marion, Intervention pour plaie du coeur. Presse médicale. 1899.

2) Kosinski bei Niedzielski, Leber Herzwunden. Kronika lecarska. Nr. 2. Ref. Jahresbericht für Chirurgie. 1899.

3) Horodynski und Maliszewski, Leber Herzwunden. Medycyna 1899. Ref. Jahresbericht für Chirurgie. 1599.

4) Carnobel, Une observation de plaie du coeur traitée par la suture. Gazette des hopitaux. 1900. Ref. Jahresbericht für Chirurgen. 1900.

5) Fontan, Plaie du coeur. Suture du ventricule gauche. Bull. et mém. de la socjété de Chirurg. de Paris. 1900.

6) Derselbe, Plaie du coeur traitée avec succès par la suture. Semaine médicale 1901. p. 395 .

7) Williams cit. bei Terrier und Reymond. Chirurgie du coeur et du péricard. Paris 1898 und bei Podrez. La Chirurgie du coeur. Revue du chirurgie. 1899. 19. Bd.

5) Walker, Deutsche Zeitschr. f. Chirurgie. 1901. 5\&. Bd.

9) Giordano, Riforma medica. 1595. Desgl. Semaine medicale. 1895. 
20. Nanu 1): Stichwunde in der Spitze des rechten Ventrikels durch Catgutnähte geschlossen. Perikard vernäht. Tod am 5. Tage in Folge jauchiger Pleuritis.

21. Lastaria 2): Herznaht nach schwerer Stichverletzung mit starkem Blutverlust, in Folge dessen Tod nach 12 Stunden.

22. Maselli ${ }^{3}$ ): Stichverletzung des linken Ventrikels. Naht unter ungünstigen äusseren Bedingungen. Heilung.

23. W a tte $\mathrm{n}^{4}$ ): Stichverletzung der rechten Brust. Wunde der rechten Pleura. $2 \mathrm{~cm}$ lange Wunde des rechten Ventrikels. Naht. JodoformgazeTamponade des Perikards. Heilung.

24. Pagenstecher ${ }^{5}$ ): $3^{1 / 2} \mathrm{~cm}$ lange Stichwunde des linken Ventrikels am 2. Tage nach der Verletzung genäht. Ueberwältigend starker Bluterguss in die Pleura, Perikardwunde verkleinert und durch die Celloidinzwirnfäden der Herznaht drainirt. Heilung.

25. Pagenstecher ${ }^{6}$ ): Stich in die Herzgegend. Freilegung des Herzens nach Ninni (Rotter). Bei Eröfinung des Herzbeutels wird das Blut darin vom Herzen zu Schaum geschlagen. Die Art. coron. sin. ist angestochen, sie wird, da sie bei der prallen Füllung des linken Ventrikels mit Schiebern nicht zu fassen ist, umstochen. Perikardlose drainirt. Tod nach 5 Tagen an eitriger Perikarditis, Pleuritis. Daneben besteht Myocarditis und Nephritis.

26. Ninni 7): Stichwunde des linken Ventrikels. Naht. Am folgenden Tage Tod.

27. Ninni ${ }^{8}$ ): Stichwunde des linken Ventrikels. Naht. Heilung.

28. Ninn ${ }^{9}$ ): Stichverletzung der rechten Brust und Pleura. $21 / 2 \mathrm{~cm}$ lange Wunde des rechten Ventrikels. Naht. Tod am 4. Tage in Folge septischer Pleuritis.

29. Mignon 10): Selbstmordversuch durch Messerstiche im Laufe einer Pneumonie. Herzverletzung. Naht. Kurz darauf schneller Tod.

30. Zulehner ${ }^{11}$ ): Stichwunde des rechten Ventrikels. Am 2. Tage

1) Nanu, Un cas de plaie du coeur, traité par la suture. Centralbl.f. Chir. 1900 ref.

2) Lastaria, Su di un caso di sutura cardiaca. Rif. medica. 1901. No. 68. Desgl. Deutsche med. Wochenschr. 1901. Literaturbeilage.

3) Maselli, Ein Fall von Herznaht. Ref. Wiener med. Presse. Nr. 9.

4) Watten, Deutsche med. Wochenschr. 1901. Nr. 37.

5) Pagenstecher, Deutsche med. Wochenschr. 1899. Nr. 32.

6) Derselbe, Ebenda. 1901. Nr. 4.

7) Ninni, Annals of Surgery. 1899. 30. Bd.

8) Derselbe, Sutur einer Wunde des Ventrikels mit glücklichem Ausgang. Rif. medica. 1901. No. á6. Ref. Münchner med. Wochenschr. 1901. Nr. ว0.

9) Derselbe, Un altra suttura del cuore per amplia ferita penetrante del seno destro. Rif. med. 1901. No. 205. Ref. Deutsche med. Wochenschr. Nr. 38. 1901. Literaturbeilage.

10) Mignon, Une observation de suture d'une plaie du coeur. Société de Chirurgie. Sem. médic. 1901. p. 142.

11) Zulehner. Zur Herznaht. Wiener klin. Wochenschr. 1901. Nr. 11. 
Herznaht, bei der die Seide durch das myokarditisch entartete Herz durchschneidet. Tod anf dem Operationstisch.

31. Nietert 1): Stichverletzung der rechten Sternalseite. Intakte Pleura. Wunde des rechten Ventrikels. Jodoformgazetamponade. Tod nach 32 Stunden an Perikarditis.

32. Nietert: Stichwunde des linken Ventrikels. Operation nach Rotter. Naht. Heilung.

33. Tummi ${ }^{2}$ ): Nicht penetrirende Stichwnnde an der Herzspitze. Naht. Heilung trotz aufgetretenen Empyems.

34. Vaughan $\left.{ }^{3}\right): 2^{1 / 2} \mathrm{~cm}$ lange Stichwunde des linken Ventrikels. Naht. Verblutungstod kurz nach beendeter Operation.

35. Rosa4): Stichwunde des linken Ventrikels. Naht. Heilung.

36. Lang o 4): Stichwunde des linken Ventrikels. Naht. Tod nach mehreren Tagen.

37. Nicolaid): Stichwunde des rechten Ventrikels. Tod wenige Stunden nach der Naht in Folge Verblutung.

38. Maselli4): Stichwunde des linken Ventrikels. Naht. Tod nach wenigen Stunden in Folge Verblutung.

39. Tuzzi4): Stichwunde des linken Ventrikels. Herznaht. Tod nach 22 Tagen in Folge eitriger Perikarditis und Empyems.

Rechne ich die 3 Barth 'schen Fälle hinzu, so sind das insgesammt 42 Fälle mit 17 Heilungen; in 25 Fällen starben die Operirten. Das macht 40,4 Proc. Heilungen gegenüber den von Fischer ${ }^{5}$ ) berechneten 10 Proc. bei nicht Operirten. Schon dieses Zahlenverhältniss wird uns zur Operation bestimmen können, umsomebr, als es sich doch bei Erweiterung unserer Erfahrungen und Vervollkommnung unserer Technik noch günstiger gestalten dürfte.

Von den 42 Fällen betraf die Verletzung 19 mal den linken, 14 mal den rechten Ventrikel, 5 mal die Herzspitze, 1 mal das linke Herzohr, 1 mal die Coronararterie, 2 mal ist der Sitz nicht angegeben. Das Ueberwiegen des linken Ventrikels bei zur Naht gelangten Verletzungen über den rechten und wiederum den Ventrikel über die Arterien erklärt sich durch die Dicke der Musculatur in den einzelnen Herzabschnitten. Eine dicke Musculatur bietet in Folge ihrer Contractionsfähigkeit und durch die Länge des durch sie verlaufenden Wundcanals sehr viel mehr Chancen zur automatischen Verschliessung

1) Nietert, Penetrating woundt of the heart with suturing of the woundt. Report of a case. Philadelphia med. News. Dec. 14.1901.

2) T'ummi bei Momburg, Veröffentlichungen aus dem Gebiete des Militärsanitätswesens. Ueber penetrirende Brustwunden und deren Behandlung. Heft 19.

3) Vaughan, A case of suture of a stabe wound of the heart. Medical News. 1901. 7. Dec.

4) Bei Nietert, s. Anm. 1.

5) Fischer l. c. 
der Wunde durch Thromben als eine dünne Musculatur. Daher sind Verletzungen des linken Ventrikels am günstigsten gestellt und gelangen am ehesten noch zur Operation; danach kommen Verletzungen des rechten Ventrikels und am ungünstigsten stehen sich Verletzungen der Vorhöfe und Aurikel, weil die Verletzten sich meist auf der Stelle verbluten. Die Verletzung der Coronararterie scheint an sich prognostisch nicht so ungünstig zu liegen, wie man früher annahm. Der Fall Pagenstecher's bestätigt, was auch die Thierversuche Bode's ergeben hatten, dass es unbeschadet der Ernährung des Herzmuskels möglich ist, eine Coronararterie zu unterbinden.

37 mal war die linke, 3 mal die rechte Pleura mit verletzt, 2 mal war die Pleura nicht verletzt.

Todesursache war $6 \mathrm{mal}$ eitrige Pleuritis, $3 \mathrm{mal}$ eitrige Perikarditis, 3 mal eitrige bezw. fibrinöse Perikarditis und Pleuritis, 1 mal Pleuritis und Pneumonie. 7 mal trat der Tod im Laufe der nächsten 24 Stunden in Folge der durch den starken Blutverlust bedingten Anämie ein, 2 mal verblutete sich der Patient unmittelbar nach beendeter Operation, 1 mal trat der Tod am 3. Tage in Folge Blutung aus einem Aste der Coronararterie ein, in 2 Fällen ist die Todesursache nich angegeben.

Von den 42 Verletzungen sind 41 Stich- und 1 Schussverletzung. Um auf die Schussverletzungen kurz einzugehen, so liegt die Seltenheit, mit der sie noch bis vor den Operateur gelangen, wohl an der Grösse der gesetzten Wunde und der dabei meist vorhandenen stärkeren Zerreissung des Klappenapparates, endlich an dem Vorhandensein einer zweiten Ein- oder Ausschussöffnung. Da diese sich an der der Brustwand abgekehrten Seite des Herzens findet, so dürfte sie überhaupt für das Anlegen einer Naht kaum zugänglich sein, eine Thatsache, an der auch im Falle Stern') die Operation nach Freilegung des Herzens scheiterte. Ausser Stern's Fall sind solche Freilegungen des Herzbeutels bezw. Herzens nach Sehussverletzungen noch 3 gemacht worden, ohne dass die Naht folgte. Bardenheuer ${ }^{2}$ ) klemmte nur die Perikardwunde ab, wagte aber noch nicht, den Herzbeutel zu eröffnen, so dass sich der Patient binnen Kurzem verblutete. Podrez ${ }^{3}$ ) eröffnete 4 Tage nach erfolgter Verletzung wegen eintretenden Fiebers und Zeichen von Infection des Herzbeutelinnern den Herzbeutel. Er fand nach Ausspülung der Auflagerungen eine $1 \mathbf{~ c m}$ lange, bereits verklebte Wunde über der Herzspitze, konnte aber trotz

1) Stern, Rotter'sche Operation. Münchner klin. Wochenschr. 1s99. Nr. 13.

2) Bardenheuer, 2 Verletzungen lebenswichtiger Organe. Münchner med. Wochenschr. 1899.

3) Podrez, l. c. 
Palpation mit beiden Händen und 8 maligem Einstechen mit einer Nadel zur Sondirung die Kugel nicht finden. Die Patientin genass vollkommen. Auf den Fall Riedel') endlich komme ich gelegentlich der Frage der Operationsmethode noch zu sprechen. Ein zweiter Fall von Herznaht nach Schussverletzung - Bufnoir, bei Momburg ${ }^{2}$ ) eitirt - ist mir nicht zugänglich geworden, auch hier starb der Patient.

Nach alledem muss wohl die jetzige Anschauung dahin gehen, dass bei Schussverletzungen des Herzens eine Indication zur Operation seltener vorliegt, und dass wir uns im Allgemeinen besser abwartend verhalten werden, zumal zahlreiche Fälle mit eingeheiltem Geschoss bekannt sind ${ }^{3}$ ).

Die Variabilität der Herzverletzungen, von der ich vorher sprach, tritt uns in den Barth'sehen 3 Fällen gleich bezüglich der Symptome entgegen, mit denen die Patienten in's Krankenhaus kommen. Während sie in den ersten beiden Fällen ganz oder theilweise benommen, völlig pulslos, mit mühsamer Athmung eingeliefert werden, vermag der 3. Verletzte auf Fragen zu antworten, der Puls ist mittelkräftig, 92 in der Minute, die Athmung ohne Beschwerden. Warum also in dem einen Falle all die Zeichen einer schweren Verletzung und im anderen nicht minder schweren so wenig bedrohliche Symptome?

Woran erkennen wir eine Herzverletzung?

Der Sitz der äusseren Wunde ist natürlich das erste, was uns an die Möglichkeit einer Verletzung des Herzens denken lassen wird, wenn andere Erscheinungen, schlechter Puls, auffallende Blässe, schlechte Athmung, Benommenheit daneben bestehen. Aber ein sicherer Schluss ist aus den Begleitsymptomen nicht zu machen, dieselben kommen ebenso gut bei jeder Verletzung der Pleura, ohne dass das Herz betheiligt ist, vor, und umgekehrt darf man den Verdacht einer Herzverletzung, auch wenn die Wunde nicht im Bereich der Herzgrenzen sitzt, nicht ausser Acht lassen. Im Barth'schen ersten Falle bestand, wie wir gesehen haben, der Verdacht der Verletzung der Bauchhöhle mit innerer Blutung, ebenso berichtet Walker in seinem 2. Falle, dass man erst nach Erweiterung der unter dem linken Rippenbogen gelegenen Wunde und nach vorsichtiger Sondirung mit dem Finger erkannt habe, dass es sich nicht um eine Verletzung der Bauch.

1) Riedel bei Terrier und Reymond. Desgl. XXVI. Congress der Deutschen Gesellschaft für Chirurgie. 1897.

2) Momburg l.c.

3) Vgl. Fischer l. c. Ferner Chirurgencongress 1902. Berlin; desgl.Ri e thus, Deutsche Zeitschr. f. Chirurgie. 67. Bd. S. 414. 
organe, sondern des Herzens handle. Es kommen eben sämmtliche Uebergänge von den allerbedrohlichsten Erscheinungen bis zur vollen Ahnungslosigkeit des Patienten von seiner Herzverletzung vor. Rose ${ }^{1}$ ) berichtet von einem Züricher Kaufmann, der eines Tages einen kurzen, stechenden Schmerz in der Brust fühlte und später eine Nähnadel vermisste, die er im Chemisette stecken gehabt hatte. Nach 3 Tagen, während deren er völlig wohl und munter gewesen war, starb er plötzlich, und die von seiner Lebensversicherungsgesellschaft bewirkte Section ergab, dass die Nähnadel im Herzen steckte und hier eine allmählich sich vergrössernde penetrirende Wunde geschaffen hatte. Der Fall beweist ferner, dass ein Schluss von der Grösse der äusseren auf die der Herzwunde auch nur bedingt zulässig ist. Es sind zahlreiche Fälle bekannt, in denen das in der Brustwand momentan fixirte verletzende Instrument in Folge der Pulsation des Herzens in diesem eine weit grössere Wunde aufriss als die äussere war.

Auch die objectiven Untersuchungsbefunde der Percussion und Auscultation sind variabel. Eine Vergrösserung der Herzdämpfung kann in Folge der Anfüllung des Herzbeutels mit Blut bestehen, kann aber auch auf einen Bluterguss in die Pleura bei intaktem Herzbeutel bezogen werden. Die sicheren Symptome einer Herzbeutelverletzung: sind allein Hämo- und Pneumoperihard, letzteres aus der gleichzeitig mitverletzten Pleura entstehend. Die Phänomene des Hämoperikards bestehen in eigenthtumlich plätschernden ("Mühlrad"-)Geräuschen, die synchron mit der Pulsation erfolgen, sowie in starker Dämpfung der Herztöne, die wie aus weiter Entfernung zu kommen scheinen. Letzterer Umstand ist insofern auffallend, als nach den Angaben Terrier's und Reymond's ${ }^{2}$, Nietert's ${ }^{3}$ ) und auch in den Barth'schen Fällen das Herz durch den Bluterguss nicht von der vorderen Brustwand abgedrängt wird, sondern stets das meiste Blut im Herzbeutel hinter dem Herzen liegend gefunden wurde. Pneumoperikard äussert sich durch tympanitischen Schall bei der Percussion; der Eintritt der Luft aus der Pleura erzeugt ferner in dem bereits mit Blut erfüllten Herzen ein eigenthümliches Brodeln, dessen Fehlen Nietert in seinem ersten Falle dadurch erklärt, dass bei ihm die Pleura unverletzt war. Dass von vornherein der Rückschluss aus dem Fehlen brodelnder Geräusche auf unverletzte Pleura nicht gemacht werden kann, beweisen die Barth'schen und zahlreiche andere Fälle mit normalem Herzbefund bei verletzter Pleura. Ein

1) Rose l. c.

2) Terrier und Reymond. Chirurgie du coeur et du pericard. Paris 1895.

3) Nietert l. c. 
solcher Rückschluss kann offenbar deswegen nicht gemacht werden, weil der Eintritt von Luft in den Herzbeutel nur unter ganz bestimmten physikalischen Bedingungen möglich ist. Ebenso weehselnd wie die physikalischen Befunde am Herzen sind die Nebenbefunde. Der Puls, der in Folge von grösserer Blutung oder in Folge Ohnmacht oder in Folge Herztamponade sehr schwach und unregelmässig sein kann, ist auch kein sicherer Rathgeber. Wo die genannten Bedingungen, wie im dritten Barth'schen Falle, fehlen, kann ein relativ guter Puls eine vielleicht verhängnissvolle Täuschung über die Schwere der Verletzung hervorrufen. In der Mehrzahl der Fälle ist der Puls allerdings in Folge der Behinderung des Herzschlages durch die im Herzbeutel allmählich zunehmende Blutmenge kaum fühlbar. Auch unsere Fälle, bei denen der Puls sofort nach Eröffnung des Herzbeutels und Entfernung des darin enthaltenen Blutes wieder fühlbar und kräftig wurde, bestätigen das häufige Vorhandensein und die Wichtigkeit dieses Befundes, auf den Rose, wie Eingangs erwähnt, zuerst aufmerksam gemacht hat.

Woher die plötzliche Ohnmacht in den meisten Fällen im Moment der Verletzung kommt, ist noch nicht genügend aufgeklärt. Die früher als Ursache angenommene Blutung kann nicht in Frage kommen, nachdem gerade Fälle mit tiefer Ohnmacht bei kaum nennenswerther Blutung beobachtet sind und ausserdem die Ohnmacht meist unmittelbar nach der Verletzung auftritt. Wahrscheinlich handelt es sich ebenso wie in den Fällen, in denen sofort der Tod nach der Verletzung eintritt (mort foudroyante), um nervöse Einflüsse. Während die einen (Frank) eine Reflexwirkung annehmen und dafür die im Herzmuskel selbst gelegenen sensiblen Nervenganglien in Anspruch nehmen, die zufällig getroffen sein sollen, wird die Ohnmacht von Anderen als eine Folge der Erschütterung des Herzens des Shoks betrachtet. Für letztere Auffassung spricht auch die Thatsache, dass diese Ohnmachten am häufigsten bei Wunden durch Feuerwaffen sowie bei Herzverletzungen in Folge stumpfer Gewalten ohne äussere Wunde beobachtet sind.

Ganz interessant ist in dieser Beziehung der von MansellMoullin ${ }^{1}$ ) veröffentlichte Fall, bei dem es sich um einen jungen Mann handelte, der bei einer Fussballpartie einen heftigen Stoss vor die Brust erhielt und ohnmächtig zusammenbrach. Die Ohnmacht hielt an und der Chirurg schloss daraus und aus der verbreiterten Herzdämpfung auf eine Herzverletzung. Er machte die Perikardo-

1) Mansell-Moullin, A case of Haemopericardium. Lancet. Clinical society of London. 1897. 
tomie, fand den Herzbeutel mit Blut angefüllt, während aus dem Herzen keine Blutung mehr stattfand. Er drainirte und der Kranke wurde völlig gesund, sodass er wieder Fussball spielen konnte. Der Fall ist darum besonders interessant, weil er uns zeigt, wie wir auch bei inneren Herzverletzungen ohne äussere Wunde mit Erfolg operiren können.

Zu den Verletzungen durch stumpfe Gewalten müssen wir übrigens wohl auch die seltenen Schussverletzungen des Herzens bei intaktem Perikard rechnen, wie sie zuerst $J_{u}$ sti $^{1}$ ) in einem Falle beschrieb. Wenigstens erscheint die Erklärung, dass derartige Verletzungen als Folge des stumpfen Aufpralles des Geschosses zu betrachten sind (Heidenreich), weniger complicirt als die andere, wonach das nur noch schwache Geschoss den Herzbeutel vor sich her in die Musculatur des Herzens hineinstülpt und der innere Blutdruck sowie die Elasticität des Herzbeutels letzteren sammt dem Geschoss wieder heraustreiben.

Auf die Frage der Sonden untersuchung brauche ich wohl nicht einzugehen, da allgemein der Standpunkt eingenommen wird, dass wir mit ihr nicht nur nichts erreichen, was die Diagnose sichern könnte, sondern eventuell durch Verdrängung eines verklebenden Coagulums oder durchsehnittenen und der Herzwunde vorgelagerten Trabekels wie im Falle Robbins ${ }^{2}$ ) eine neue letale Blutung hervorbringen können. Die einzige erlaubte Sondirung ist die unter Be-obachtung strengster Asepsis und nach eventueller Erweiterung der Wunde mit dem Finger vorgenommene, die auch in einzelnen Fällen, wie im zweiten Walker'schen und ersten Barth'schen Falle die Diagnose auf Herzverletzung sicherte. Im Uebrigen giebt es, abgesehen von der von Rotter ${ }^{3}$ ) als Voroperation angegebenen schichtweisen Erweiterung der Wunde, auf die wir bei Besprechung der Operationsmethoden noch zurückkommen, kein Mittel, aus dem wir mit unbedingter Sicherheit die Diagnose einer Herzverletzung stellen können. Der Schluss auf eine solche wird immer nur aus einzelnen Symptomen zusammengenommen mit mehr oder minder grosser Wahrscheinlichkeit gemacht werden können, und dies ist wohl auch mit ein Grund dafür, dass wir so verhältnissmässig spät mit einer Chirurgie am Herzen begonnen haben.

Weswegen operiren wir jetzt an Stelle der früheren exspectiven

1) Justi, Ein Fall von schwerer Herzverletzung ohne Verletzung des Herzbeutels. Deutsche med. Wochenschr. 1900. Nr. 50.

2) Robbins, Medical Record. New-York. Nov. 1880.

3) Rotter l. c. 
Therapie? Was können wir mit einer Operation nützen und welchen Schaden können wir thun?

Wie schon Fingangs erwähnt, forderten zunächst zur Operation alle Fälle auf, bei denen der Patient ohne eine solche sicher zu Grunde geht; das sind die Fälle mit starker primärer Blutung und die mit Herztamponade. Sie geben beide die absolute Indication zur Operation ab. Mit dieser Indication können wir uns aber nach unseren jetzigen Erfahrungen nicht begnügen. Denn wir werden einmal bei der eben beschriebenen Schwierigkeit in der Erkennung der Herzverletzungen stets dic Möglichkeit eines plötzlichen Todes auch bei an sich harmlos aussehenden Fällen in's Auge fassen müssen und ferner bietet die Gefalr secundärer Blutungen eine weitere Indication. Derartige secundäre Blutungen können in verschiedenen Stadien der Reconvalescenz eintreten und sind natïrlich bei der meist schon bestehenden Anämie des Patienten von sehr ungünstiger Prognose. Die häufigsten secundären Blutungen treten auf, wenn der Kranke sich von seiner anfänglichen Ohnmacht wieder erholt hat, Durch letztere war eine allgemeine Blutdruckerniedrigung geschaffen worden, die die Bildung eines verstopfenden Coagulums begünstigte. Wenn nun der Herzschlag wieder kräftiger zu schlagen beginnt bevor dieses Coagulum die nöthige Festigkeit erlangt hat, so wird es leicht hinweggeschwemmt und die nun folgende Blutung, die automatisch nicht melr zum Stehen kommt, ist meist letal. Dann aber müssen secundäre Blutungen auch später stets gewärtigt werden. Dic einen erfolgen beim ersten Aufstehen oder der ersten stärkeren Anstrengung des Patienten aus den Synechien, die sich bei spontan geheilten Herzverletzungen in Folge Verklebung der beiden Blätter des Ilerzbeutels viel zahlreicher bilden wie bei genähten Wunden, bei denen der Inhalt des Herzbeutels bei dessen Eröffnung entleert wird. Terrier und Reymond ${ }^{\prime}$ ) beschreiben einen solchen Fall, bei dem der Patient nach einer ersten grösseren Anstrengung unter den Zeichen von Herztamponade ohnmächtig zusammenbrach und sich, bevor die Operation gemacht werden konnte, aus den gerissenen Synechien verblutet hatte. Die andere Art secundärer späterer Blutungen erfolgt aus der gerissenen Narbe. Da nämlich die Wundheilung am Herzen nicht durch Regeneration des Herzmuskels, sondern durch directe Narbenbildung vor sich geht, so entsteht eine viel weniger widerstandsfähige Narbe als wenn die Ränder der Wunde direct durch Naht mit einander vereinigt wären. Die Folge ist, dass

1) Terrier und Reymond l. c. 
es leicht zu aneurysmatischen Hervorwölbungen der Narbe kommt, sowie Patient schwere Arbeit beginnt, und dass die Gefahr des Platzens dieser Herzaneurysmen und damit einer tödtlichen Blutung noch nach Jahren für den Kranken besteht. Die Gefahren der Operation an und für sich sind dem gegenüber verhältnissmässig gering. Die Freilegung des Herzens ist schnell und mit wenig Blutverlust ausführbar, und auch die Operationen am Ierzen selber sind nach den bisherigen Erfahrungen gewiss nicht leicht, aber doch jedenfalls viel weniger gefährlich als man erwartet hatte. Ich komme auf das Verhalten des Herzens bei der Naht erst an späterer Stelle zu sprechen und begnüge mich hier, nur zu erwähnen, dass sich das Herz im Allgemeinen allen Operationen gegenüber sehr tolerant erwiesen hat. Auch die durch die Narkose bedingte Gefahr ist nicht gross, da man meist wohl mit sehr wenig Chloroform auskommen dürfte und in vielen Fällen - so Nietert, Barth u. s. w. - ohne Narkose operiren konnte. Endlich ist auch die Gefahr einer Infection der Herzwunde a priori nicht gross, da wir nach allen Erfahrungen, namentlich denen der modernen Kriegschirurgie !) diese Wunden im Allgemeinen als primär aseptisch ansehen müssen. Die Zeugstïcke werden, da es sich fast stets um Stichverletzungen handelt, durchschnitten und gelangen nicht in die Wunde und der Schmutz der Waffe streift sich wohl grösstentheils an der äusseren Haut und den Weichtheilen $a b$; jedenfalls sind die Wunden im Allgemeinen frei von pathogenen Keimen. Pagenstecher stellt darum mit Recht den Satz auf, dass man, ebenso wie man heutzutage bei Verdacht einer Darmverletzung die Bauchhöhle eröffne, ebenso bei Verdacht einer Herzverletzung den Herzbeutel eröffnen solle. Die Hauptgefahr droht dem Operirten, wie meine obige Statistik ergiebt, von Seiten der Pleura, deren eitrige Entzündung 10 mal unter 2ら Fällen - entweder allein oder combinirt mit eitriger Pericarditis - den Tod herbeiführte. In Folge eines durch die Operation selber bedingten unglücklichen Zwischenfalles siud bisher keine Verluste entstanden.

Bevor wir auf die Ausführung der Operation selber eingehen, will ich noch kurz die von Rotter zuerst an Stelle der Sondenuntersuchung empfohlene Voroperation besprechen. Sie besteht in dem schichtweisen Erweitern der Wunde und ist zur Sicherung der Diagnose von Einzelnen, so in dem einen Barth'schen Falle ausgeführt worden. Man kann dadurch leicht bis zur Herzwunde vordringen und andererseits bei nicht vorliegender Herzverletzung für

1) Korsch, Kreg schirurgische Erfahrungen ans dem griecl.-tïrk. Kriege. Desgl. Küttner aus dem Transvaalkrieg. 
guten Abfluss der Wundsecrete sorgen. Ganz interessant ist in dieser Hinsicht der Fall Williams'). Es handelte sich um einen jungen Mann, der einen Degenstich in die linke Brust erhalten hatte. Zunächst wurde operativ nicht eingeschritten, als sich aber Angstgefühl, Athembeschwerden und eine Blutung einstellten, wurde die Wunde schichtweise erweitert. Man merkte nach Unterbindung der Mammaria, aus der die Blutung stammte, eine Perikardwunde von $3 \mathrm{~cm}$ und, zwischen den aufgehobenen Rändern dieser Wunde hindurchsehend, eine $3 \mathrm{~mm}$ lange Herzwunde des rechten Ventrikels. Da diese aber offenbar nicht penetrirend war und nicht blutete, hielt man es für überflüssig sie zu nähen, vernähte das Perikard nach Entfernung der Blutcoagula aus demselben mit Catgut und der Kranke genass völlig. In ähnlicher Weise machte auch Dalton ${ }^{2}$ ) eine erfolgreiche Naht des Perikards, zu dem er durch schichtweises Erweitern der Wunde gekommen war.

Eine Herzwunde selbst auf diese Weise ohne Resection von Rippen oder Brustbein zu nähen, dürfte freilich nicht möglich sein. Zur Herznaht bedarf es der offenen Freilegung des Herzens und selbst die Fälle, wo schon nach Resection einer Rippe die Herznaht gelang, wie bei Rehn und Maliszewski, dürften zu den Seltenheiten gehören und sich nur durch Kleinheit und sehr günstigen Sitz der Wunde erklären lassen.

Im Allgemeinen kann man 3 Methoden unterscheiden, die zur Freilegung des Herzens theils an der Leiche erprobt und beschrieben, theils in praxi ausgeführt sind. Während die ersten beiden die Freilegung des Herzens durch Bildung eines osteoplastischen Lappens und zwar entweder extrapleural - unter Vermeidung der Eröffnung der Pleura - oder transpleural - durch die Pleura durch zum Herzbeutel gelangend - bewirken wollen, folgt die dritte Methode dem Wundcanal auf dem Wege zum Herzen, je nach Bedürfniss dabei vom Brustbein oder den Rippen resecirend.

Wenden wir uns zur ersten dieser Methoden, 'der extrapleuralen, so finden wir sie in verschiedenen Modificationen von $W e h r^{3}$ ), Rydygiert), Pagenstecher, Napalkow ${ }^{5}$ ) u. A. beschrieben.

1) Williams, Blessure du coeur, suture du pericarde, guérison. New-York medical record. 1897.

2) Dalton citirt bei Terrier und Reymond.

3) Wehr, Eine neue Methode der Brustkorberöffnung. Verhandlg. der deutschen Gesellschait für Chirurgie. 1599.

4) Rydygier l. e.

5) Napalkow, Dissertat. Moskau. Ref. Centralbl. f. Chirurgie. 1900. 
Sie alle bilden einen länglichen mit der Basis nach rechts gelegenen Hantmuskelknochenlappen aus einem Theil des Brustbeins im Zusammenhange mit mehr oder weniger langen Stücken linker Rippenknorpel, lösen den Lappen subperiostal los und klappen ihn nach rechts herüber. Sie henutzen damit also die von $\mathrm{Jamain}^{1}$ ) so bezeichnete chondrosternale Herzregion, d. h. diejenige Partie des Brustkorbes, durch die hindurch man zum IIerzen gelangen kann, ohne die Pleura zu verletzen. Die Methode hat nun aber nur dann einen Zweck und Vorzug, wenn einmal die Pleura bis dahin noch nicht eröffnet ist und wenn man dies ferner vor Eröffnung des Brustkorbes feststellen kann. Beides ist nicht oder doch nur äusserst selten der Fall. Von dèn 42 Fällen, die ich gesammelt habe, ist die Pleura, wie schon erwähnt, nur zweimal - im Falle Pagenstecher und $\mathrm{Nietert} \mathrm{-} \mathrm{unverletzt.} \mathrm{In} \mathrm{den} \mathrm{übrigen} \mathrm{Fällen} \mathrm{ist} \mathrm{sie} \mathrm{durch} \mathrm{das} \mathrm{die}$ Herzwunde setzende Instrument mit verletzt. Man würde also unter diesen Verhältnissen nicht nur zwecklos die Pleura geschont, sondern vor Allem kostbare Zeit verloren haben und sogar in den meisten Fällen nachber noch in die Nothwendigkeit versetzt sein, die Pleura nachträglich breit öffnen zu müssen, um dem darin enthaltenen Blut Abfluss zu schaffen. Dazu kommt endlich noch ein weiterer Umstand, der bei nicht eröffneter Pleura von Pagenstecher und vor ihm von Riedel beobachtet war. Bei Beiden wurde im Moment der Eröffnung des Herzbeutels das darin enthaltene Blut durch die Pulsation des Herzens zu Schaum geschlagen, wodurch die Operation ausserordentlich erschwert, ja im Falle Riedel unmöglich gemacht wurde. Pagenstecher erklärt dies Zuschaumschlagen durch den plötzlichen Lufteintritt durch den breit geöffneten ILerzbeutel, im Gegensatz zu dem langsameren und allmählichen Lufteintritt aus der gleichzeitig verletzten Pleura.

Alle diese Gründe sprechen ebenso gegen die extrapleurale Freilegung des Herzens wie die Thatsache, dass sie meines Wissens iì praxi noch nicht versueht ist. Sie kann meines Erachtens nur in den seltenen Fällen in Frage kommen, wo volle Zeit zur Operation und die Wahrscheinlichkeit einer bisher unverletzten Pleura vorhanden ist, also z. B. in Fällen, wo der Arzt erst Stunden nach erfolgter Verletzung hinzugerufen wird und bei gutem Allgemeinbefinden des Kranken operiren kann, mit andern Worten, wenn die Blutung sicher steht und augenblickliche Gefahren nicht mehr zu befürchten sind. Transpleurale Methoden, um zum Herzbeutel zu gelangen, sind

1) Jamain bei Terrier und Reymond. 
von Rotter, Parozzani, Pamoni und Del Vecehio angegeben bezw. angewandt. Die ersten 3 machen einfache osteoplastische Lappen, die sie nach rechts, Pamoni nach unten herumklappen, Del Vecchio schlägt einen Thürflügelschnitt vor, bei dem die Flügel nach oben und nach unten auseinandergeklappt werden. Gut bewährt hat sich von ihnen die Rotter'sche Methode, die bereits dreimal - von Pagenstecher, Stern und Nietert - angewandt worden ist. Alle 3 loben die schnelle Ausführbarkeit der Operation, die mir auch bei meinen Versuchen an der Leiche ebenso wie die gute Freilegung des Herzens durch sie aufgefallen ist. Immer aber, wie es Rotter von seiner Methode verlangt, wird sie trotzdem nicht anwendbar sein und zwar in den seltenen Fällen von rechtsseitiger Pleuraverletzung nicht. Bisher waren allerdings erst 2 solcher Fälle - Watten und Ninni - bekannt, der erste Barth'sche würde der 3. Fall sein. In allen drei Malen wurde auch dementsprechend die Eröffnung des Brustkorbes von rechts her gemacht.

Die 3. Methode endlich ist von Giordano ${ }^{\prime}$ angegeben und besteht, wie schon erwähnt darin, auf dem Wege des Wundcanals das Herz freizulegen und dabei an Knochentheilen das fortzunehmen resp. osteoplastisch zuräckzuklappen, was die Herznaht behindert. Es ist die Methode, die, weil natürlich immer anwendbar, auch praktisch bisher am meisten verwerthet ist, und nach der auch Barth in seinen Fällen operirt hat. Nietert, der in seinem ersten Falle bei intakter Pleura nach Giordano operirte, gelang es sogar gut, dieselbe unverletzt zu erhalten. Die Nachtheile der Methode bestehen darin, dass man keine glatte Wunde erhält und nach der Heilung gerade die vor dem Herzen liegende Partie des Brustkorbes theilweise ihres knöchernen Schutzes beraubt ist. Wie jedoch der Barth'sche Patient beweist, der sich völlig wohl befindet, trotzdem man das Herz durch die alte Operationsnarbe hindurch regelrecht palpiren kann, sind diese Nachtheile nicht gross.

Bezüglich der weiteren Operation möchte ich nur noch auf einzelne Punkte kurz eingehen; zunächst auf die Herznaht selber und das Verhalten des Herzens dabei. Während wohl bei den früheren Chirurgen hauptsächlich die Scheu, am lebenden, pulsirenden Herzen zu operiren, der Grund dafür war, dass man erst so spät zu einer Chirurgie des Herzens schritt, hat sich das Herz nach allen jetzigen Erfahrungen Operationen gegentuber sehr tolerant erwiesen. Wie auch Barth betont, wird die Herzaction an sich durch die Eröffnung des

1) Giordano, La chirurgia del Pericardio. 
Herzbeutels nicht beeinflusst und nur vorübergehend unterbrochen, wenn es mit Instrumenten berührt wurde. Was man dem Herzen alles bieten kann beweist der vorher geschilderte Fall Podrez.

Um die erste und schwierigste Naht anzulegen, wurde das Herz entweder von hinten her mit der Hand gegen das Brustbein gedrängt (Watten) oder direct mit der Hand gefasst ( $\mathrm{Nanu}$ und Carnobel). Nietert bediente sich dazu zweier Kocherzangen, Barth breiter stumpfer Hacken. Nachdem erst einmal eine Naht hindurchgelegt war, wurde das Herz daran vorgezogen und liess sich leicht in die Wunde einstellen. Die Schwierigkeit der ersten Naht beruhte nicht allein auf der Pulsation, sondern auch auf der durch die Athmung bedingten Zwerchfellbewegung. Elsb erg ${ }^{1}$ ) empfiehlt nach seinen Erfahrungen an Thieren die Inspiration für die Durchführung der Nadel, weil bei ihr das Herz nach vorn gedrängt wird, während es bei der Exspiration zurïcksinkt. Er beobachtete ferner bei seinen Versuchen eine Reaction des Herzens nur beim Stich durch das Epi- und Endokard, während das Herz nicht reagirte, so lange die Nadel im Myokard war. Beim Anziehen der Nähte beobachtete Watten eine deutliche Verlangsamung der Herzschlages, während Rehn und Barth sogar kurzen Stillstand desselben sehen. Was die Naht selbst anbetrifft, so wurde in den meisten Fällen die Knopfnaht an Stelle der fortlaufenden gewählt. Wenn letztere auch als schneller gehend von Fontan empfohlen wird, so ist die Knopfnaht doch wohl sicherer. Gleichfalls der besseren Haltbarkeit wegen wurde fast stets als Material die Seide dem eventuell zu schnell resorbirten Catgut vorgezogen. Bei der Naht wurde nur das Epi- und Myokard gefasst. Einmal ist dann die Blutung aus den Stichcanälen geringer, so lange das Endokard unverletzt ist, ferner wird eine Gerinnung des Blutes, wie sie stets an Fremdkörpern eintritt, innerhalb des Herzens und damit die Gefahr der Loslösung eines Thrombus vermieden. Endlich schneidet nach Elsb e rg auch eine durchgreifende Naht leichter durch als eine Myokardnaht. Solches Durchschneiden der Naht ist bisher erst zweimal von Walker und Zulehner - beobachtet worden. Beide Male handelte es sich um einen völlig entarteten Herzmuskel, bei dem in W alker's Falle die Naht, wie schon erwähnt, erst gelang, nachdem das Perikard mitgefasst und ein Tupfer eingebunden war.

Ebenfalls um ein Durchschneiden der Nähte zu vermeiden, empfiehlt Elsberg, sie in der Diastole zu knoten. Er beobachtete, dass die in der Systole geknoteten Nähte in der Diastole durch.

1) Elsberg, Ueber Herzwunden und Herznaht, nebst histologischen Untersuchungen über die Heiling der Herzwunden. Centralbl. f. Chirurgie. 1899. Nr. 43. 
schnitten. Freilich dürfte es nicht immer leicht sein, den richtigen Zeitpunkt abzupassen. Barth in seinem ersten Falle und vor ihm Watte $n$ betonen die 'Unmöglichkeit, die einzelnen Herzphasen $z u$ unterscheiden, solange das Herz gefasst war. Uebrigens erklären sich wohl aus demselben Grunde zum Theil auch die so verschiedenartigen Angaben über die Blutung aus der Wunde in den einzelnen Herzphasen. Man findet bei ganz gleichgearteten Herzwunden in dem einen Falle die Angabe einer systolischen, im anderen einer diastolischen, im dritten Falle einer permanenten Blutung. Um ganz kurz darauf einzugehen, so besteht allerdings ein Lnterschied zwischen kleinen und grossen Wunden der Ventrikel derart, dass man bei kleinen meist nur diastolische, bei grossen systolische oder permanente Blutung findet. Dieser Umstand erklärt sich sehr leicht dadurch, dass bei kleinen Wunden bei der Systole sich der Herzmuskel so zusammenzieht, dass der ganze Wandeanal verlegt wird, was bei grösseren nicht mehr möglich ist. Besonders günstig liegen aus diesem Grunde schräg durch die Musculatur verlaufende Wunden. Auf den Gegensatz zwischen Ventrikeln und Atrien bezüglich der Stärke der Blutung bin ich schon vorber eingegangen.

Zum Schluss möchte ich noch eine, wie mir scheint, sehr wichtige Frage besprechen, nämlich die: sollen wir nach vollendeter Herznaht auch den Herzbeutel primär vernähen oder sollen wir einen $\Lambda$ bfluss offen halten, indem wir Drains oder Tampons einlegen oder, wie Pagenstecher, die Fäden der Naht zur Perikardwunde hinaus. leiten. Letzterer bezeichnet die primäre Naht direct als "einen Fehler, der gelegentlich den tödtlichen Ausgang herbeiführen könne", und auch Watte $n$ hält sie für nicht ungefährlich. Im Gegensatz hierzu kam Barth nach den Erfahrungen seines ersten Falles, der mit Perikarditis endigte, dazu, bei seinen späteren beiden Fällen primär zu nähen. Ich habe daraufhin aus meinen IIerzfällen eine Zusammenstellung gemacht und darin die Angaben über primäre Naht oder Drainage des Herzbeutels in Beziehung zu dem Ausgang des Patienten bezw. dem Sectionsbefund gesetzt.

Da bei vielen Fällen keine diesbezügliche Angabe gemacht war und andererseits die Fälle irrelevant waren, bei denen der Tod nicht in Folge Parikarditis eintrat, so blieben mir nur 16 verwerthbare Fälle, deren Ergebniss ich hier mittheile.

In 9 Fällen wurde der Herzbeutel primär vernält resp. mit dem Herzen zusammen (Rehn, Giordano) vernäht. Davon trat Heilung in 6 Fällen (Rehn, Parozzani, Fontan 2 und Barth 2) ein; dreimal erfolgte der Tod an eitriger Pleuritis und zwar am 5., 19. 
und 21. Tage, nachdem bedrohliche Erscheinungen von Seiten des Herzens nicht aufgetreten waren und bei der Section eine gute und und reactionslose Heilung der Herzwunde constatirt werden konnte.

In 7 Fällen wurde der Herzbeutel offen gelassen resp. nur theilweise vernäht und drainirt. Davon erfolgte in 2 Fällen (Watten, Pagenstecher) Heilung, in 5 Fällen dagegen wurde bei der Section fibrinöse oder eitrige Parikarditis gefunden (Malis zewski, Walker, Pagenstecher, Nietert, Barth), d. h. es trat gerade das Entgegengesetzte von dem ein, was man mit der Drainage beabsichtigt hatte. Die Grïnde für dieses auffallende Ergebniss lassen sich wohl leicht genug erklären. Abgesehen von der schnelleren Heilung der primären Naht wird die Infectionsgefahr durch die Drainage nicht verringert, sondern vermehrt. Wenn die Herzstichverletzungen, wie wir annehmen, als primär aseptisch betrachtet werden müssen, so ist die Infectionsgefahr a priori gering und bleibt es, wenn die Herzund die Ilerzbeutelwunde schnell geschlossen wird. Anders aber, wenn drainirt wird, weil dann die Gefahr vorhanden ist, dass der Tampon und damit die Herzwunde von aussen resp. aus dèr Pleurawunde inficirt werden können. Ebenso lässt die Thatsache, dass bei primärer Vernähung nie eine Secretverhaltung im Herzbeutel beobachtet ist, darauf schliessen, dass diese seröse Secretion, wie sie Watten z. B. hinter dem Tampon beobachtete, nicht unwahrscheinlich erst durch die Reibung des IIerzens an dem Fremdkörper entstanden war. Hierzu kommt aber als wesentlichster Grund, der für die primäre Naht spricht, noch eins: Es ist in Folge der Drainage oder Tamponade des Herzbeutels ganz unvermeidlich, dass es zu stärkeren Synechien und Verwachsungen zwischen dem Herzen und dem Herzbeutel kommt und sie fordern eine solche ausserordentliche Mehrarbeit des Herzmuskels, dass dieser ihr auf die Dauer nicht ge. wachsen ist. Es kommt ebenso wie bei den perikarditischen Verwachsungen rheumatischen oder tuberculösen Ursprungs Anfangs zu einer Degeneration der oberflächlichen Schichten des Ierzens und später zur secundären Atrophie und Dilatation des Herzens, die unter schweren Circulationsstörungen nach Jahr und Tag das Ende herbeiführen.

Die Schlüsse, die ich aus dem Vorausgegangenen ziehe, sind folgende: 1. Bei dem Verdacht auf Herzverletzung durch Stichwaffen ist die Operation, auch ohne vitale Indication zur Vermeidung späterer Schädigungen (Herzaneurysmen) geboten. Schussverletzungen des Herzens sind in der Regel exspectativ zu behandeln. 2. Die 
einzige ungefährliche Methode zur Sicherung der Diagnose ist die schichtweise Erweiterung der Wunde. 3. Die extrapleurale Methode zur Freilegung des Herzens kommt selten praktisch in Frage, da die Freilegung unbedingt mit Benutzung der ursprünglichen Wunde geschehen muss und diese erfahrungsgemäss fast stets die Pleura mit eröffnet. 4. Die Naht wird am zweckmässigsten als Knopfnaht mit nicht durchgreifenden Fäden angelegt. Die Knotung der Fäden erfolgt in der Diastole. 5. Die Herzbeutelwunde ist in der Regel durch primäre Naht zu behandeln. Die Tamponade sollte nur bei bestimmter Indicationsstellung (Infection des Herzbeutels, unstillbare Blutung) angewandt werden. 